\title{
Incipient fault detection of chiller based on improved CVA
}

\author{
Qu Jiehui ${ }^{1,2}$, Zhang Hanyuan ${ }^{1,2, *}$, Zhang Guiqing ${ }^{1,2}$, Chen Hao ${ }^{1,2}$ \\ 1. School of Information and Electrical Engineering, Shandong Jianzhu University, Jinan, 250101, China \\ 2. Shandong Key Laboratory of Intelligent Buildings Technology, Jinan, 250101, China
}

\begin{abstract}
The chiller plays an important role for providing comfort environment. Once, the incipient faults are missed, they may develop to be fatal faults and further lead to equipment damage and casualties. Nevertheless, the incipient fault in the running process of the chiller are easily neglected in noise. Moreover, the running variables of the chiller have dynamic characteristics, and each process variable is correlated with each other in each process, and a certain variable is interrelated at different times. To tackle these problems, we develop an improved canonical variable analysis (ICVA) method to detect the incipient fault in chiller units with significant dynamic characteristics. In the proposed method, the exponentially weighted moving average (EWMA) is first applied to filter the data. Then the canonical variable analysis is used to detect the fault. In this paper, ASHRAE RP-1043 experimental data are used to verify the proposed method. Simulation results show that compared with traditional CVA method, ICVA method has a higher fault detection rate for incipient fault.
\end{abstract}

\section{Introduction}

With the fast development of the economy and society, the requirements of people for comfort are higher and higher. Hence, in order to provide the comfort environment, HVAC systems are widely used in various fields, such as medical treatment, commerce, and construction. However, if the chiller of the HVAC system fails, it will not only shorten the life of the equipment, but also increase nearly $15 \%-20 \%$ of energy consumption [1] Therefore, it is of great significance to research the chiller fault detection technology, aiming at maintaining indoor comfort, reducing equipment wear and tear, and further saving energy.

During the working process of the chiller, the amplitude of some faults is incipient, resulting to the changes of them are indistinguishable and easily covered by noise. Most of the fatal faults develop from the deterioration of incipient fault. Therefore, to avoid system damage and accidents, timely and accurately detection such incipient faults are conducive and successfully attract many attentions of researchers. Gong et al. [2] proposed an improved CNNs-SVM method for the detection of incipient fault. In this method, a combination of $1 \times 1$ hyperconvolutional layer and global mean pooling layer are used to replace the traditional CNN structure for fault detection, and good results are obtained. Cai et al. [3] proposed a weighted probability kernel principal component analysis method to detect incipient fault based on kernel principal component analysis. The Kullback Leibler divergence component is used to describe the difference in the degree of fault information, and the corresponding weights are assigned to strengthen the detection of incipient fault. $\mathrm{Li}$ et al. [4] combined principal component analysis with support vector data description, established support vector description space model in residual space of principal component analysis, and used distance based monitoring information for fault detection, and achieved good results. Nevertheless, the above-mentioned literature only considers the detection of incipient fault, and does not consider the dynamic characteristics of the system in the working process.

In addition to incipient fault, there is cross-correlation and self-correlation between process variables and at different times of a variable, that is, dynamic characteristics. Aiming at the fault detection of dynamic process. Qi et al. [5] proposed an improved multi-channel principal component analysis (MPCA) method for fault detection of dynamic process by using time-varying covariance calculation statistics. Liu et al. [6] proposed a block kernel principal component analysis method. By using the kernel matrix updated with time instead of the fixed kernel matrix for the principal component model, the dynamic process detection performance of kernel principal component analysis is enhanced and the detection rate is improved. Zhang et al. [7] applied local space regularized to extract the slow features from fault data, and then combined it with independent component analysis to achieve the real-time fault detection, and verified the effectiveness through simulations. $\mathrm{Gu}$ et al. [8] proposed slow feature principal component analysis method by combining principal component analysis with slow feature analysis. Although the above documents consider the dynamic characteristics of system, the

*Corresponding author: Zhang Hanyuan; E-mail: zhanghanyuan18@sdjzu.edu.cn 
problem which the incipient fault are easily covered by noise is less considered into, leading to the lower detection rate for such incipient fault.

For the dynamic characteristics of chillers, this paper proposes an improved canonical variable analysis (ICVA) method to improve the detection rate of incipient fault. In the proposed ICVA method, considering the chiller process measuring data containing noise and noise will hide small amplitude of incipient fault, exponential weighted moving average (EWMA) is first used to eliminate noise in measured data highlight incipient fault information. Furthermore, considering the dynamic performance of the chiller significantly, the canonical variable analysis (CVA) is employed to mime the dynamics of the chiller based on the output matrix of EWMA, the low-dimensional characteristic information of process data is extracted and monitoring statistics are constructed to improve the performance of incipient fault detection of chiller. Finally, the effectiveness of the proposed method is verified on ASHRAE RP-1043 experimental dataset.

\section{Improved CVA (ICVA) method}

\subsection{Explanatory weighted moving average}

In the early stage of the incipient fault of the chiller, the extent of the fault is usually not easy to be detected, but the gradual evolution of the incipient fault will cause great harm. In order to solve the problem of incipient fault amplitude and being easily submerged by noise in the operation process of water chiller, EWMA method is used to eliminate the noise in the data of water chiller and emphasize the fault information. The core idea of EWMA is to use the operation data of the chiller at the current time and the forecast data of the previous time to construct the forecast data of the current time, which includes both the real observation data of the current time and the data information of the previous time, according to a certain weight value.

The formula $z \sim N\left(\mu, \sigma^{2}\right)$ indicates the random variable, where $\mu$ is the mean and $\sigma^{2}$ is the variance. In the random variable, if the actual observed value of the chiller at time $t$ is $Z_{i}$, then the EWMA model construction value at time $t$ is:

$$
x_{t}=\lambda z_{t}+(1-\lambda) x_{t-1}
$$

\subsection{Canonical variable analysis}

As mentioned above, the chiller has significant dynamic characteristics. In order to further improve the detection ability of incipient fault of the chiller, CVA is adopted in this paper to further deal with the operating variables of the chiller. The traditional method can not decompose the dynamic characteristics of the operating variables of the chiller well. Therefore, this paper further applies CVA method to EWMA filtered data to solve the dynamic characteristics of chiller variables. Canonical variable analysis is a linear dimensionality reduction technique widely used in multivariate statistical methods. It was first applied to fault detection by Negiz and Cinar in 1997 [9]. In the process of fault diagnosis of chiller, it needs to expand the dimension of input and output matrix of chiller to cover the historical data set and future data set of chiller in the process, maximize the correlation between the historical data set and future data set, and extract the main information of the process. Considering the temporal correlation of variables, at a specific sampling time $t$, the observed vector $x_{t} \in R$ extends forward $p$ steps at time $t$ to form the past observed variable $x_{p, t}$, and extends $t$ steps after $t$ to form the future observed variable $x_{f, t}$.

The past and future Hankel matrix is defined as $Y_{p}=\left[\begin{array}{llll}y_{p, p+1} & y_{p, p+2} & \cdots & y_{p, p+N}\end{array}\right] \in R^{m p \times N}$ $Y_{f}=\left[\begin{array}{llll}y_{f, p+1} & y_{f, p+2} & \cdots & y_{f, p+N}\end{array}\right] \in R^{n p \times N}$. In a process with $m$ observation vectors and $M$ observation values for each variable, the last element of the observation variable $y_{p, p+1}$ in the above equation can be known as $y_{1}$, according to equation 2 . The last element of the observed variable $y_{f, p+N}$ in the future can be known as $y_{1}$ according to Equation 3. The column dimension of Hankel matrix is $N=m-2 p+1$. The covariance and cross-covariance matrix of historical matrix $Y_{p}$ and future matrix $Y_{f}$ are $\sum_{p p}=Y_{P} Y_{P}{ }^{T} /(N-1)$, $\sum_{f f}=Y_{f} Y_{f}^{T} /(N-1), \quad \sum_{p f}=Y_{P} Y_{f}^{T} /(N-1)$.

Given $u 1=\sum_{f f}^{-1 / 2} a, u 2=\sum_{p p}^{-1 / 2} b$, CVA problem can be described as follows

$$
\begin{aligned}
& \max _{u_{1}, u_{2}} u_{1}^{T}\left(\sum_{f f}^{-1 / 2} \sum_{f p} \sum_{p p}^{-1 / 2}\right) u_{2} \\
& \text { s.t. } \quad u_{1}^{T} u_{1}=1 ; u_{2}^{T} u_{2}=1
\end{aligned}
$$

The above optimization problem can be solved by SVD decomposition of the HanKel matrix $H$.

$$
H=\sum_{f f}^{-1 / 2} \sum_{f p} \sum_{p p}^{-1 / 2}=U \Delta V^{T}
$$

where $U$ and $V$ are orthogonal matrices, $\Delta$ is the descending order of eigenvalues of Hankel matrix, and the typical variables are $Z_{r}$,

$$
z_{r}=\left[\begin{array}{c}
b_{1}{ }^{T} \\
b_{2}{ }^{T} \\
\vdots \\
b_{m p}{ }^{T}
\end{array}\right] y_{p, r}=V^{T} \sum_{p p}^{-1 / 2} y_{p, r}=J y_{p, r}
$$

where $J=V^{T} \sum_{P P}^{-1 / 2}$ is the transformation matrix that converts the historical measurement value of the chiller into the typical variable space. The typical variable space is composed of the main space and the remaining space. The dimension of the main space is determined by the number of dominant singular values. The main space $x_{f} \in R^{n}$ determined by the first $\mathrm{n}$ singular values can represent the main characteristics of the typical variable space. The remaining eigenvalues determine the 
remaining space $d_{r} \in R^{m p-n}$. The value of $n$ is set in advance. Therefore, the typical variable space can be expressed as:

$$
z_{r}=J_{p, r}=\left[\left[J_{x} y_{p, r}^{T}\right] d_{r}^{T}\right]^{T}=\left[\begin{array}{ll}
x_{r}^{T} & d_{r}^{T}
\end{array}\right]^{T}
$$

The state variable is not only part of the typical variable, but also a linear combination of the past observation vectors.

$$
x_{r}=J_{x} y_{p, r}=V_{x}^{T} \sum_{p p}^{-1 / 2} y_{p, r}
$$

where $V_{x}$ represents the first $x$ column of $V$.

On the basis of obtaining the CVA state variable, the state process of the chiller is monitored, and the collected data is transformed into meaningful statistics. According to the reality, a certain threshold is added to the statistics. If a statistic exceeds the threshold, a fault will be considered to have occurred.

The $T_{S}^{2}$ statistics of CVA are as follows:

$$
T_{s}^{2}=Y_{p}(t)^{T} J_{x}^{T} J_{x} Y_{p}(t)
$$

The $T r^{2}$ statistics of CVA are as follows:

$$
T_{r}^{2}=Y_{p}(t)^{T} J_{q}^{T} J_{q} Y_{p}(t)
$$

The SPE statistics of CVA are as follows:

$$
S P E=Y_{p, t}{ }^{T}\left(I-J_{x}{ }^{T} J_{x}\right)\left(I-J_{x} J_{x}{ }^{T}\right) Y_{p, t}
$$

\section{Incipient fault detection strategy of chiller based on ICVA}

In order to improve the detection rate of incipient fault of chiller with significant dynamic characteristics, an improved canonical variable analysis fault detection method for chiller is proposed. The specific strategies are as follows: In order to eliminate the noise in the process data of the chiller and highlight the incipient fault features, the EWMA method is firstly used to filter the process data of the chiller. EWMA method adds the measured data at the current moment and the forecast data at the previous moment according to certain weights to construct the forecast data at the current moment, so that the calculated forecast data contains both the data information at the current moment and the data information at the previous moment. Then, CVA is applied to the data matrix output from EWMA to solve the significant dynamic characteristics of the chiller. At the same time, $T_{S}^{2}, T r^{2}$ and SPE statistics constructed by low dimensional characteristic information are extracted to incipient fault.

The fault detection method of chiller based on ICVA proposed in this paper includes two parts: offline training and online detection. Fig. 1 is a flow chart of the detection method. The main steps of the two sections are briefly described below.

\section{Offline training}

(1) The observation variable data under normal working conditions are selected from the historical data of the chiller, and EWMA method is used to eliminate the influence of noise on the original data and highlight the incipient fault features.

(2) The data is normalized, and the mean and variance of each variable are zero and one, so as to eliminate the influence of dimension in data analysis.

(3) Hankel matrices of historical variables and future variables are constructed to calculate self-covariance and cross-covariance, and singular value decomposition is performed to extract key features.

(4) The statistical control limits of TS2, Tr2 and SPE are calculated.

\section{Online testing}

(1) Standardize the data of online detection.

(2) The data collected at time $\mathrm{k}$ is used to construct the past vector $\mathrm{pk}$.

(3) Calculate statistics TS2, Tr2 and SPE at time k and compare them with their control limits. If any statistic exceeds the limit, it indicates that fault has occurred.

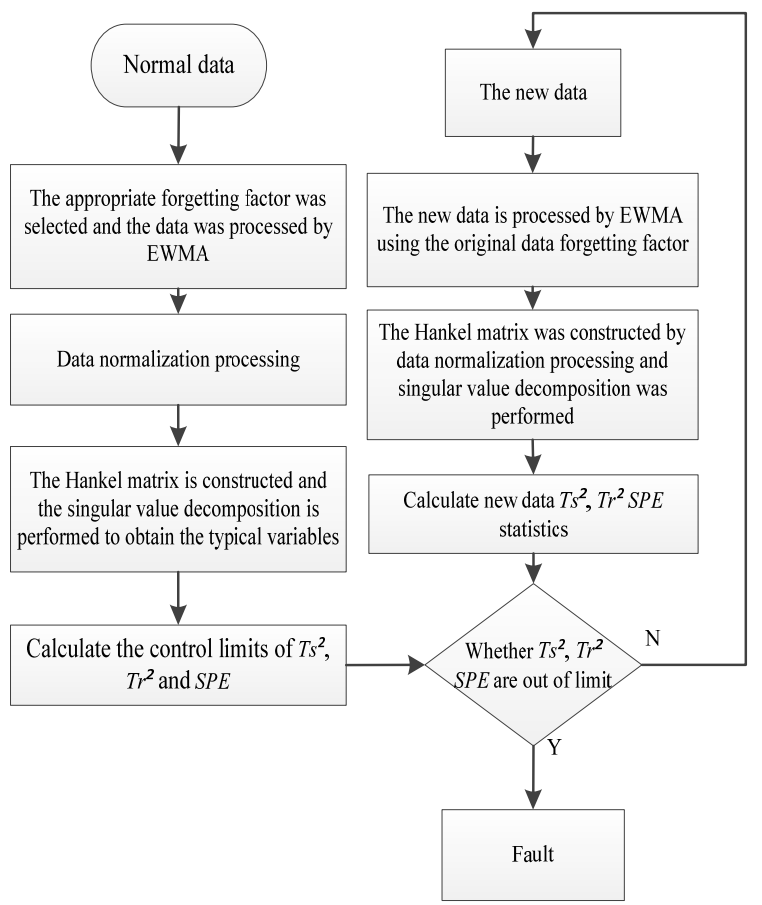

Fig. 1 ICVA fault detection method flow chart

\section{Experimental verification}

In order to verify the fault detection performance of the proposed method, sample data is selected from ASHRAE RP-1043 chiller fault simulation experiment. This experiment uses a 90t centrifugal chiller. Through a specially designed test bed, the typical failures of seven kinds of chiller are artificially simulated. The seven faults are as follows: over refrigerant (F1), evaporator water flow reduction (F2), condenser water flow reduction (F3), non-condensable gas in the refrigerant (F4), condenser fouling (F5), insufficient refrigerant charge or leakage (F6) and excess oil (F7). Four different severity levels, SL1-SL4, are considered for each failure. The least severe is rated as SL1 and the most severe as SL4. The fault degree of SL1 is relatively light. In this paper, the incipient fault degree of SL1 is considered as incipient fault. In the experiment, the incipient fault of 7 kinds of faults in the simulation experiment of the chiller are taken as examples for verification. 


\subsection{The simulation results}

According to the data collected by the ASHRAE RP-1043 project, each sample contains 64 characteristic variables. In this paper, 12 variables such as pressure, temperature and power of water chiller are selected. For each variable, 500 samples under normal state are selected as training data, and another 500 samples are selected as test data, among which the first 200 groups are normal data. From the moment of 201, incipient fault is added. The forgetting factor of EWMA model is selected as 0.2 , and seven kinds of faults of the chiller are detected by MATLAB simulation. Taking insufficient condenser water flow (F3) as examples, the experimental data with the lowest fault degree is selected to represent

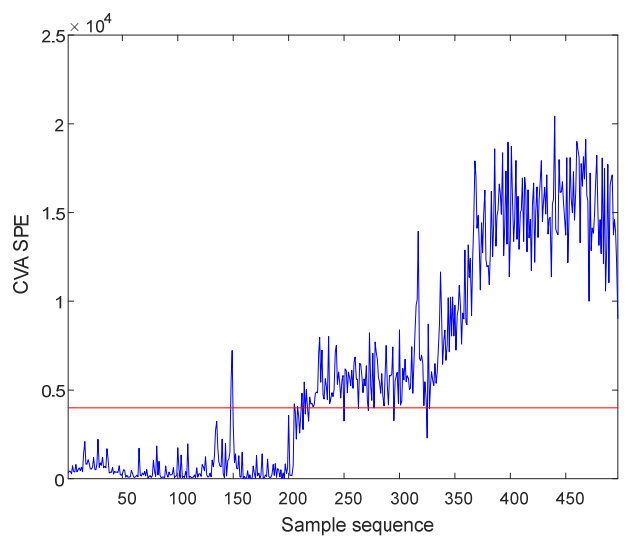

(a)Monitoring chart of fault F3 by SPE statistic of CVA

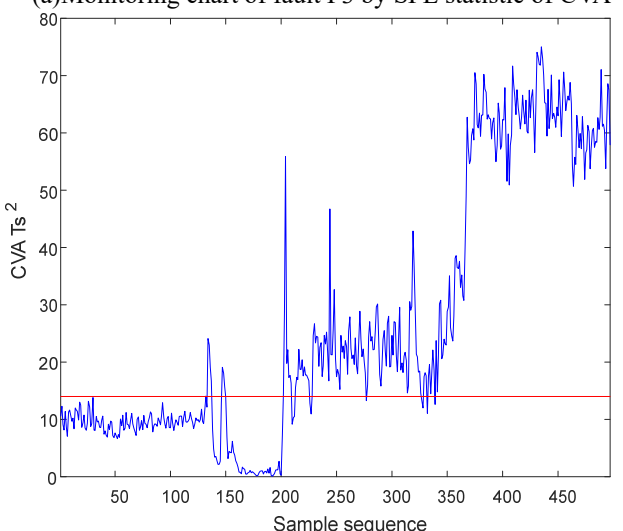

(c)Monitoring chart of fault F3 by $T s^{2}$ statistic of CVA

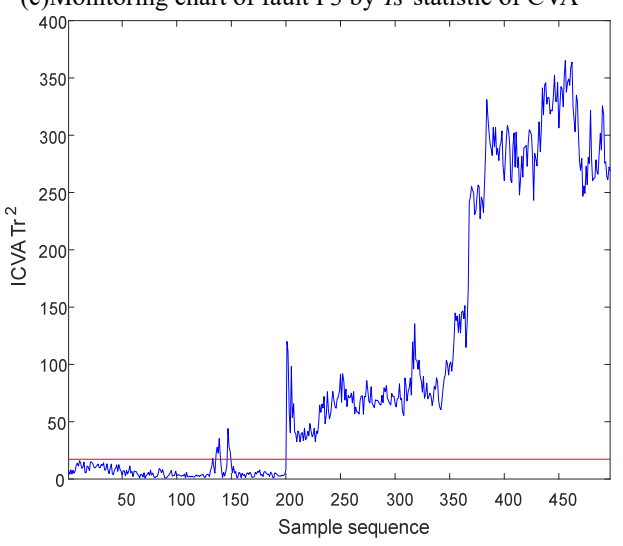

(e)Monitoring chart of fault F3 by $T r^{2}$ statistic of ICVA the incipient fault. In addition, Gaussian white noise is added to simulate the measurement noise in the field. The PCA model and CVA model are selected as comparison methods to verify the effectiveness of the proposed method in detecting incipient fault of chillers.

Taking fault F3 (10\%) as an example, the fault detection diagrams of the three methods are shown in the Fig. 2. The monitoring results of CVA and ICVA methods for fault F3 are shown in Fig. 2. Compared with the fault detection results in Fig. 2(a) (c), the ICVA method in Fig. 2(d), (e) and (f) achieves the best fault detection effect. The SPE, $T r^{2}$ and $T s^{2}$ statistics of ICVA all detect fault at 201, and the fault detection rates are all $100 \%$.

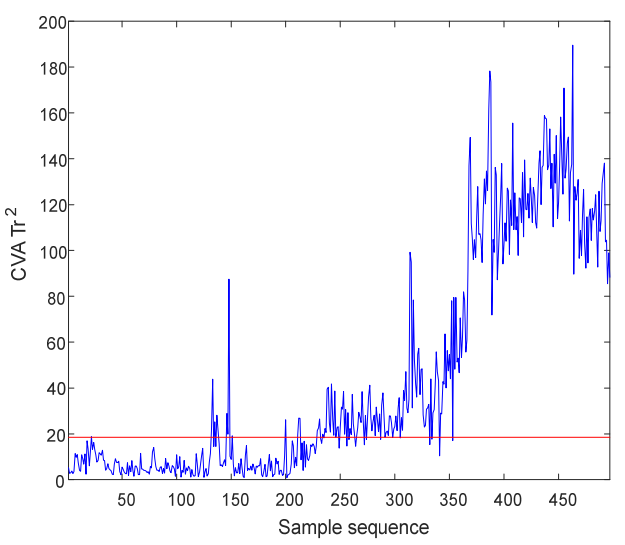

(b) Monitoring chart of fault F3 by $T r^{2}$ statistic of CVA

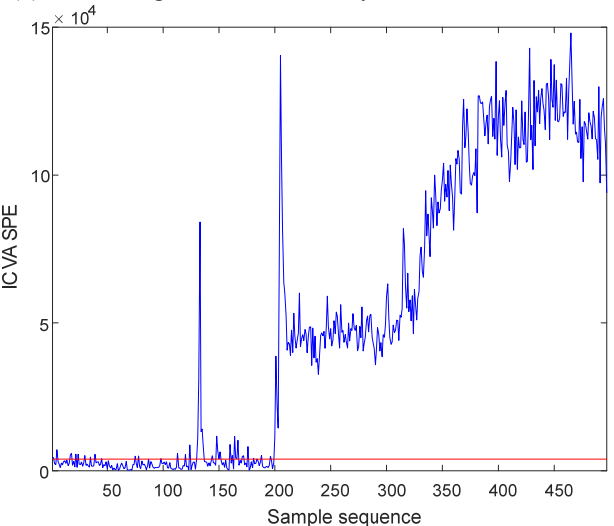

(d) Monitoring chart of fault F3 by SPE statistic of ICVA

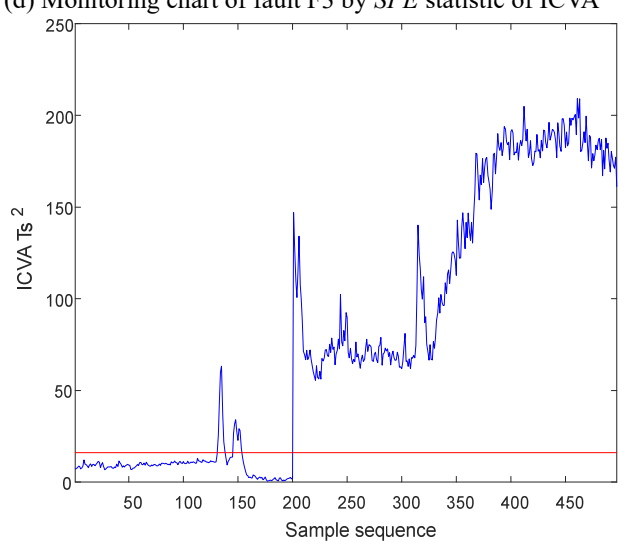

(f) Monitoring chart of fault F3 by $T s^{2}$ statistic of ICVA

Fig. 2 Monitoring diagram of fault F3 in the operation process of chiller by two fault detection methods 
Table 1 Comparison of fault detection rates of the two fault detection methods

\begin{tabular}{ccccccc}
\hline \multirow{2}{*}{$\begin{array}{c}\text { The fault } \\
\text { number }\end{array}$} & \multicolumn{3}{c}{ CVA } & \multicolumn{3}{c}{ ICVA } \\
\cline { 2 - 7 } & $T s^{2}$ & $T r^{2}$ & $S P E$ & $T s^{2}$ & $T r 2$ & $S P E$ \\
\hline F1 & 95 & 93 & 89 & 100 & 100 & 100 \\
F2 & 96 & 87 & 100 & 100 & 100 & 100 \\
F3 & 94 & 80 & 93 & 100 & 100 & 100 \\
F4 & 96 & 96 & 98 & 100 & 100 & 100 \\
F5 & 99 & 99 & 88 & 100 & 100 & 100 \\
F6 & 87 & 80 & 92 & 100 & 100 & 100 \\
F7 & 90 & 93 & 77 & 100 & 100 & 100 \\
\hline
\end{tabular}

The fault detection rates of the two fault detection methods for 7 types of fault modes are shown in Table 1 . As can be seen from Table 1, the fault detection rates of $S P E, T r^{2}$ and $T s^{2}$ statistics of CVA method are all more than $80 \%$, and the fault detection rate of F2 is $100 \%$. The detection rate of the three statistics of ICVA method proposed in this paper all reach $100 \%$. In conclusion, the results confirm that the ICVA based method has superior fault detection performance compared with the CVA based method.

\section{Conclusion}

In this paper, a new ICVA method is proposed to improve the detection rate of incipient fault of chiller with significant dynamic characteristics. In this method, EWMA is used to preprocess the operation data of the chiller. Then, CVA is used to process the significant dynamic characteristics of the chiller. The fault detection effect of ICVA method proposed in this paper is verified in ASHRAE RP-1043 dataset. Compared with CVA methods, the simulation results show that ICVA method has a higher detection rate for incipient fault of chiller.

\section{Acknowledgments}

This research was partly funded by the Shandong Provincial Major Science and Technology Innovation Project (2019JZZY010115), the National Natural Science Foundation of China (62003191), the Natural Science Foundation of Shandong Province (ZR2020QF072), and the Doctoral Research Foundation of Shandong Jianzhu University (XNBS1821).

\section{References}

1. Yang L, Yan H, Lam J C. Thermal comfort and building energy consumption implications - A review. Applied Energy, 2014, 115: 164-173.

2. Gong $\mathrm{W}$ F, Chen $\mathrm{H}$, Zhang $\mathrm{M}$ L, Zhang $\mathrm{Z} \mathrm{H}$. Intelligent Fault Diagnosis Method for Motor Bearing Based on Deep Learning. Chinese Journal of Scientific Instrument, 2020, 41(01): 195-205.
3. Cai P P, Deng X G, Cao Y P, et al. Small fault detection in nonlinear chemical process based on WPRKPCA. Chemical Engineering Progress, 2019, 038(012): Scientific Instrument, 2020, 41(01): 195-205.

4. Li G N, Hu Y P, Chen H X, et al. An improved fault detection method for incipient centrifugal chiller faults using the PCA-R-SVDD algorithm. 2016, 116: 104-113.

5. Qi Y S, Wang $P$, Gao X X, et al. An Improved MPCA Based Method for Batch Process Monitoring and Fault Diagnosis. Chemical Engineering Journal, 2009, 060(011): 2838-2846.

6. Liu C Y, Yu C M. The Fault Diagnosis Based on Improved KPCA.Computer Measurement and Control, 2016, 24(010): 36-38.

7. Zhang Z B, Wang Z L, Wang X. Fault Detection Method Based on Orthogonal Local Chronic Feature. Journal of Tsinghua University: Science and Technology, 2020(8): 693-700.

8. Gu S M, Liu Y L, Zhang N. Fault Diagnosis in Tennessee Eastman Process Using Slow Feature Principal Component Analysis. 2016, 9(1): 49-61.

9. Negiz A, Cinar A. Statistical monitoring of multivariable dynamic processes with state-space models. Aiche Journal, 2010, 43(8):2002-2020. 